\title{
ON MATIJASEVITCH'S NONTRADITIONAL APPROACH TO SEARCH PROBLEMS
}

\author{
Andreas BLASS * \\ Mathematics Department, University of Michigan, Ann Arbor, MI 48109, U.S.A. \\ Yuri GUREVICH * * \\ Electrical Engineering and Computer Science Department, University of Michigan, Ann Arbor, MI 48109, U.S.A. \\ Communicated by David Gries \\ Received 4 April 1988 \\ Revised 2 September 1988
}

Keywords: Nontraditional approach, search problems, NP-complete problems, satisfiability problem, differential equations, randomization

\section{Introduction}

In [2], Yuri Matijasevitch, famous for completing the solution of Hilbert's tenth problem suggested three very nontraditional methods to solve the search version of the well-known NP-complete problem 3-SAT: Find values of propositional variables $p_{1}, \ldots, p_{n}$ satisfying a given formula

$A=\bigwedge_{m}\left(p_{f(m, 1)} \vee p_{f(m, 2)} \vee p_{f(m, 3)}\right)$

where $1 \leqslant f(m, k) \leqslant 2 n$ and $p_{n+i}=\neg p_{i}$ for all $i \leqslant n$. He wrote: "A unifying feature of the methods is that they are inspired by real phenomena in nature. The phenomena were only the starting point for reflection; they give a convenient language, but the author does not propose to realize the corresponding physical processes. We speak only about solving the corresponding differential equations."

- The work of the first author was partially supported by NSF Grant DMS 85-01752.

* The work of the second author was partially supported by NSF Grant DCR85-03275. Also, during the final stage of the work the second author was with Stanford University and IBM Almaden Research Center (on a sabbatical leave from the University of Michigan).
We offer here our critical reaction, pointing out some apparently major problems with the suggested methods. In spite of the criticism, the spirit of nontraditional methods appeals to us.

For the reader's convenience, the reaction is interleaved with descriptions of the methods.

\section{Method I: In vitro}

\section{Description of the method}

Reactions among $2 n$ chemicals $M_{1}, \ldots, M_{2 n}$ in a solution lead to formation of precipitates; namely, precipitates result from

- every pair $M_{i}$ and $M_{n+i}$,

- every triple $M_{f(m, 1)}, M_{f(m, 2)}$ and $M_{f(m, 3)}$.

The concentrations $c_{1}(t), \ldots, c_{2 n}(t)$ of chemicals $M_{1}, \ldots, M_{2 n}$ satisfy differential equations:

$c_{i}^{\prime}=-\alpha c_{i}^{\lambda} c_{n+i}^{\lambda}-\beta \sum\left(c_{f(m, 1)}^{\mu} c_{f(m, 2)}^{\mu} c_{f(m, 3)}^{\mu}\right)$

where the sum is over all $m$ such that $i=f(m, k)$ for some $k$, and $c_{2 n+i}=c_{i}$, and parameters $\alpha, \beta, \lambda, \mu$ are positive.

The system tends to a limit equilibrium $c_{1}(\infty), \ldots, c_{2 n}(\infty)$ with $c_{i}(\infty) c_{n+i}(\infty)=0$. Call an equilibrium degenerate if fewer than $n$ chemicals remain. Every equilibrium gives a partial assign- 
ment to variables $p_{1}, \ldots, p_{n}: p_{i}$ is true if $c_{i}(\infty)=$ $0 \neq c_{n+i}(\infty)$, and $p_{i}$ is false if $c_{i}(\infty) \neq 0=$ $c_{n+i}(\infty)$. If the equilibrium is nondegenerate then this is a full assignment that satisfies $A$.

A state of the system is determined by the current concentrations $c_{i}$. The initial states are chosen randomly from the unit hypercube. Matijasevitch asks whether the event [the equilibrium is degenerate] comprising the initial states that lead to degenerate equilibria, is of measure zero.

\section{Comments}

Unfortunately, the method does not work even for 2-SAT; we presume that if the $m$ th clause contains only two literals $p_{f(m, 1)}$ and $p_{f(m, 2)}$, then it contributes a summand $-\beta c_{f(m, 1)}^{\mu} c_{f(m, 2)}^{\mu}$ to the corresponding differential equations. The formula

$B=\bigwedge\left\{\left(\neg p_{i} \vee p_{j}\right): j=i+1\right.$, or else $i=n$ and $j=1\}$

has exactly two satisfying truth assignments, one with all $p_{1}, \ldots, p_{n}$ true and one with all $p_{1}, \ldots, p_{n}$ false. But the corresponding chemical reaction has many stable equilibria. In the cyclic ordering

$\neg p_{1}, p_{2}, \neg p_{2}, p_{3}, \ldots, \neg p_{n-1}, p_{n}, \neg p_{n}, p_{1}$,

any initial distribution that has at least one zero concentration between every two non-zero ones is an equilibrium, i.e., will not change with time. If, furthermore, there are never more than two consecutive zero concentrations (in the cyclic order above), then the equilibrium is stable in the following sense: any sufficiently close initial condition leads to the same pattern of zero and non-zero concentrations (but not, of course, to the same numerical values of the non-zero ones, so we are dealing not with a stable state but with a stable manifold). The number of stable equilibria increases exponentially with $n$, and it is reasonable to expect that $\operatorname{Pr}[$ the equilibrium is degenerate] quickly increases with $n$. We prove next that this expectation is correct.

In the next lemma, $A$ is the instance of 3-SAT exhibited in the introduction or a similar formula with some clauses of length 2 . In particular, the lemma applies to $B$.
1.1. Lemma. Suppose that $p_{j}$ appears as a literal in every clause of $A$ that contains $p_{i}$ as a literal and that $c_{j}(0)>c_{j}(0)+c_{n+i}(0)$. Then $c_{i}(\infty)>c_{j}(\infty)+$ $c_{n+i}(\infty)$.

Proof. It suffices to prove that the derivative $\left(c_{i}-\right.$ $\left.c_{j}-c_{n+i}\right)^{\prime}$ is never negative. Rewrite the derivative using the differential equations for $c_{i}, c_{j}$ and $c_{n+i}$. The term $-\alpha c_{i}^{\lambda} c_{n+i}^{\lambda}$ arising from $c_{i}^{\prime}$ is cancelled by the corresponding term arising from $c_{n+i}^{\prime}$. The other terms arising from $c_{i}^{\prime}$, those involving $\beta$, are cancelled by corresponding $\beta$ terms arising from $c_{j}^{\prime}$. After these cancellations, only nonnegative terms remain.

1.2. Lemma. If reals $x_{1}, x_{2}, x_{3}$ are randomly and independently chosen from the real interval $[0,1]$ then $\operatorname{Pr}\left[x_{2}>x_{1}+x_{3}\right]=\frac{1}{6}$.

Proof. In the unit cube of all possible triples $\left(x_{1}, x_{2}, x_{3}\right)$, the triples that satisfy $x_{2}>x_{1}+x_{3}$ form a tetrahedron whose volume is easily computed to be $\frac{1}{6}$.

Let the event [ $A$ fails] comprise the initial states leading to degenerate equilibria such that the corresponding partial assignments cannot be extended to a total assignment satisfying $A$.

1.3. Theorem. As $n$ increases, $\operatorname{Pr}[B$ fails $]$ approaches 1 exponentially fast (relative to $n$ ).

Proof. Let

$$
\begin{array}{r}
q_{1}, \ldots, q_{2 n}=\left(\neg p_{1}, p_{2}, \neg p_{2}, p_{3}, \ldots, \neg p_{n-1},\right. \\
\left.p_{n}, \neg p_{n}, p_{1}\right)
\end{array}
$$

and let $d_{j}$ be the concentration of the chemical corresponding to $q_{j}$. To simplify the exposition, we suppose that $n$ is divisible by 3 . Split the interval $[1 . .2 n]$ into 6-tuples [1..6], [7..12], [13..18], and so on.

By Lemma 1.2 and the independence of random variables $d_{j}$, the probability that some

$d_{6 k+2}(0)>d_{6 k+1}(0)+d_{6 k+3}(0)$

equals $1-\left(\frac{5}{6}\right)^{n / 3}$. Similarly, the probability that some 
$d_{6 l+5}(0)>d_{6 l+4}(0)+d_{6 l+6}(0)$

equals $1-\left(\frac{5}{6}\right)^{n / 3}$. By the independence, the probability that there are $k$ and $l$ such that

$d_{6 k+2}(0)>d_{6 k+1}(0)+d_{6 k+3}(0)$

and

$d_{61+5}(0)>d_{61+4}(0)+d_{6 t+6}(0)$

equals $\left(1-\left(\frac{5}{6}\right)^{n / 3}\right)^{2}$. By Lemma 1.1, the probability that some $d_{6 k+2}(\infty)>0$ and some $d_{6 l+5}(\infty)>$ 0 is at least $\left(1-\left(\frac{5}{6}\right)^{n / 3}\right)^{2}$. But every $d_{6 k+2}$ is some $c_{i}$ with $i \leqslant n$, and every $d_{6 l+5}$ is some $c_{n+i}$ with $i \leqslant n$. Hence, every event $\left[d_{6 k+2}(\infty)>0 \wedge\right.$ $d_{6 l+k}(\infty)>0$ ] implies the event [ $B$ fails].

The exponential character of convergence is important in the light of an attractive conjecture that $\mathrm{NP}=\mathrm{R}$. Recall that a language $L$ over some alphabet $\Sigma$ is in $\mathrm{R}$ if there exist a deterministic Turing machine $M$ and polynomials $g, h$ such that for every $\Sigma$-string $s$ the following are equivalent:

- $s \in L$,

- there is a binary string $t$ of length $h(|s|)$ such that $M$ accepts the pair $(s, t)$ within time $g(|s|)$, and

- for at least one half of all binary strings $t$ of length $h(|s|), M$ accepts the pair $(s, t)$ within time $g(|s|)$.

It is clear that $R \subset N P$ and that $N P=R$ if 3-SAT belongs to $\mathrm{R}$. If $\operatorname{Pr}$ [the equilibrium is not degenerate] exceeds $|A|^{-k}$ for some constant $k$ and all (sufficiently long) formulas $A$, then repeating the experiment only polynomially many times yields $\operatorname{Pr}$ [in at least one run, the equilibrium is not degenerate] $>\frac{3}{4}$; this would imply that 3 -SAT belongs to $\mathrm{R}$. But then, in the case of $B, \operatorname{Pr}[$ the equilibrium is not degenerate] exceeds $n^{-2 k}$ for sufficiently large $n$ (because $|B|=\mathrm{O}(n \log n)=$ $\left.\mathrm{O}\left(n^{2}\right)\right)$, which contradicts Theorem 1.3.

In Theorem 1.3, we dealt, for simplicity, with an instance $B$ of 2-SAT (rather than 3-SAT) and we assumed a corresponding modification of Matijasevitch's differential equations. Let us exhibit similar difficulties in the originally proposed context of 3-SAT. (One of our referees felt that this is unnecessary, but a proponent of Matijase- vitch's method may feel differently.) Define $B^{\prime}$ to be the instance of 3-SAT obtained from $B$ by replacing each clause $\left(\neg p_{i} \vee p_{j}\right)$ with

$\left(\neg p_{i} \vee p_{j} \vee q\right) \wedge\left(\neg p_{i} \vee p_{j} \vee \neg q\right)$,

where $q$ is a new variable. The proof of Theorem 1.3 can be repeated to establish that $\operatorname{Pr}\left[B^{\prime}\right.$ fails] approaches 1 exponentially fast.

One may argue that the failure of Method I for the specific formulas $B$ and $B^{\prime}$ does not exclude the possibility that the method works for "most" formulas. But the traditional methods, like backtracking, are known to solve 3-SAT in expected polynomial time with respect to many natural probability distributions; see, for example, [3]. The formula $B$ was chosen as a simple illustration of fairly general phenomena. It is still possible that Method I gives a good heuristics for an important subclass of 3-SAT; it is up to the proponents of the method to exhibit such a subclass.

\section{Method II: "Creation"}

\section{Description of the method}

In a $k$-dimensional space, there are $c_{i}$ atoms of an element $a_{i}$ where $1 \leqslant i \leqslant 2 n$. The following gravitational forces act:

(1) If $|i-j| \neq n$ then an atom of $a_{i}$ and an atom of $a_{j}$ attract each other with force $F(r)$ where $r$ is the distance between them; otherwise they repel each other with force $G(r)$.

(2) If $i=f(m, 1), j=f(m, 2)$ and $k=f(m, 3)$ for some $m$, then three atoms of $a_{i}, a_{j}$ and $a_{k}$ respectively repel with forces $H\left(r_{i, j}, r_{j, k}, r_{k, i}\right)$, $H\left(r_{j, k}, r_{k, i}, r_{i, j}\right), H\left(r_{k, i}, r_{i, j}, r_{j, k}\right)$ where $r_{i, j}, r_{j, k}$, $r_{k, i}$ are the respective distances.

Forces $F, G, H$ decrease to zero at infinity and increase to infinity when atoms draw together in an unbounded way. In addition, "the force of the friction on vacuum" may exist; it may depend on velocities and/or higher derivatives. A "Newton's law" holds: the acceleration (or a higher derivative) is proportional to the sum of acting forces. (One of the referees suggests that the three forces in (2) should lie in the plane of the three atoms and should sum to zero, so that Newton's third law holds.) 
In the evolution of the system, planets-gatherings of atoms with only attracting forces-may form. A planet with atoms of $n$ elements gives an assignment

$p_{i} \leftrightarrow$ [the planet has no atoms of $a_{i}$ ]

to $p_{1}, \ldots, p_{n}$ that satisfies the formula $A$. Matijasevitch asks whether it is possible to use the arbitrariness in the choice of "physical laws" to create a model where, for not very big quantities $c_{i}$, some $n$-element planets will form in a relatively short time provided the formula $A$ is satisfiable.

\section{Comments}

Since only one planet of the right size is needed, it seems reasonable to expect that such a planet will form if $A$ is satisfiable and there is a sufficiently big number of atoms of each kind. But then it may take too long to decide whether the final state has a large enough planet.

A space of finite measure seems more appropriate for the "experiment". One possibility is to consider the unit hypercube and stipulate that whenever an atom leaves the hypercube it immediately reappears from the opposite side.

In the case of formula $B$, there are only two sets of $n$ elements $a_{i}$ and exponentially many sets of fewer than $n$ elements $a_{i}$ such that the corresponding planets repel any atom of any kind that does not exist on the planet. It seems unlikely that for not very big quantities $c_{i}$, some $n$-element planets will form in a relatively short time.

The relative magnitudes of forces are very important. If attractive forces are comparable to repulsive then there may be stable gatherings with some repulsive forces (pseudo-planets); a few repelling atoms may be kept on a pseudo-planet by overwhelming attractive forces. But with strong repulsive forces, a planet can have pieces knocked loose by near-collisions with atoms that it repels.

\section{Method III: Resonance}

\section{Description}

There are $2 n+1$ pendulums $P_{0}, \ldots, P_{2 n}$ which are one-dimensional oscillators satisfying the dif- ferential equation $x^{\prime \prime}=-a x$ in the absence of outside forces. The following additional forces, depending on velocities, influence pendulums $P_{1}, \ldots, P_{2 n}$.

- Braking forces act on $P_{i}$ and $P_{n+i}$ when they swing in the same direction, and accelerating forces act on them the rest of the time.

- Braking forces act on $P_{f(m, 1)}, P_{f(m, 2)}, P_{f(m, 3)}$ when they all swing in the direction of $P_{0}$ and accelerating forces act the rest of the time.

Given a satisfying assignment for $A$, swing $P_{i}$ in phase (resp. out of phase) with $P_{0}$ if $p_{i}$ is false (resp. true); here $1 \leqslant i \leqslant 2 n$. In this initial moment, only accelerating forces influence pendulums $P_{1}, \ldots, P_{2 n}$, and one may expect that also in the future most of the time there will be no braking forces and, out of two pendulums $P_{i}$ and $P_{n+i}$, one will be almost always in phase with $P_{0}$ and the other will be almost always out of phase. Is it true that if formula $A$ is satisfiable then the system will arrive at a similar regime from almost any initial state?

It is possible, writes Matijasevitch, to introduce forces of friction and parametric pumping [1].

\section{Comments}

The third method is different; the idea seems to be to avoid stable configurations of wrong kinds. Again, the relative magnitudes of forces are of great importance. If braking forces are comparable to accelerating ones then unwanted configurations may resonate, with the braking forces being dominated by accelerating ones. If braking forces are much stronger than accelerating then they may bring the system to a halt.

The method introduces many arbitrary parameters to determine the exact forces. Since this makes analysis very difficult, we consider a greatly simplified model, which we hope preserves some of the spirit of Matijasevitch's proposal.

We represent the motion of the pendulum corresponding to a literal $q$ of the formula $A$ by a number $x_{q}$ whose absolute value is the amplitude of the oscillation, with $x_{q}<0$ (resp. $x_{q}>0$ ) if the pendulum is in phase (resp. out of phase) with the yard-stick pendulum $P_{0}$. (We pretend that intermediate phases do not occur.) As a further simplification, we consider a simple discrete-time ana- 
log. We suppose that every conjunct in $A$ is a 2-clause.

At each time step, each $x_{q}$ is updated by getting a number of summands. One summand is contributed by the pair $(q, r)$ where $r$ is the opposite of $q$, i.e., either $q$ is a variable and $r=\neg q$ or else $r$ is a variable and $q=\neg r$. This summand is

if $x_{q} x_{r}>0$ then $-\alpha \operatorname{sig}\left(x_{q}\right)$ else $\alpha^{\prime} \operatorname{sig}\left(x_{q}\right)$.

In addition, every clause $q \vee r$ contributes a summand

if $x_{q}<0$ and $x_{r}<0$ then $\beta$ else $\beta^{\prime} \operatorname{sig}\left(x_{q}\right)$.

Here $\alpha, \alpha^{\prime}, \beta, \beta^{\prime}$ are positive reals. At each moment $t$, the system defines the following assignments $s_{A}(t)$ to the propositional variables: A variable $p$ is true if and only if $x_{p}>0$. We suppose that the initial values $x_{q}(0)$ are drawn at random from some real interval $[-\nu . . \nu]$ with $\nu$ exceeding any of the four numbers $\alpha, \alpha^{\prime}, \beta, \beta^{\prime}$.

Let $h$ be the least integer s.t. $2 \alpha^{\prime}+(2 h+1) \beta^{\prime}$ $\geqslant \beta$, and let $C$ be the extension of the formula $B$ by means of clauses $q \vee q^{j}$ where $q$ ranges over the $2 n$ literals of $B$ and $1 \leqslant j \leqslant h$ and each $q^{j}$ is a new propositional variable (the total number of variables in $C$ is $n+2 n h$ ).

3.1. Theorem. The probability that the assignment $s_{C}(t)$ does not satisfy $C$ for any $t$ whatsoever approaches 1 exponentially fast (relative to $n$ ).

Proof. For each variable $p=p_{i}$ of $B$, let $x_{i}, y_{i}, x_{i, j}, x_{i, j}^{\prime}, y_{i, j}, y_{i, j}^{\prime}$ abbreviate quantities $x$ with subscripts $p, \neg p, \quad p^{j}, \neg\left(p^{j}\right), \quad(\neg p)^{j}$, $\neg\left((\neg p)^{\prime}\right)$ respectively. As in the proof of Theorem 1.3 , we assume for simplicity that $n$ is divisible by 3. Break the sequence $x_{1}, y_{1}, x_{2}, y_{2}, \ldots, x_{n}, y_{n}$ into 6-tuples

$\left[x_{3 u+1}, y_{3 u+1}, x_{3 u+2}, y_{3 u+2}, x_{3 u+3}, y_{3 u+3}\right]$

3.2. Lemma. (1) Suppose that $y_{3 u+1}(t)>0$, each $x_{3 u+1, j}(t)>0$, each $x_{3 u+1, j}^{\prime}(t)<0$, each $y_{3 u+1, j}(t)$ $>0$, each $y_{3 u+1, j}^{\prime}(t)<0$ and $y_{3 u+1}(t)>\alpha+$ $x_{3 u+1}(t)$ if $t=0$. Then the same holds holds for every $t$.

(2) Suppose that $x_{3 u+3}(t)>0$ and each $x_{3 u+3, j}(t)>0$, each $x_{3 u+3, j}^{\prime}(t)<0$, each $y_{3 u+3, j}(t)$
$>0$, each $y_{3 u+3, j}^{\prime}(t)<0$ and $x_{3 u+3}(t)>\alpha+$ $y_{3 u+3}(t)$ if $t=0$. Then the same holds for every $t$.

Proof. By symmetry, it suffices to prove only the first statement. Without loss of generality, $u=0$. It is easy to see that each $x_{1, j}(t)>0$, each $x_{1, j}^{\prime}(t)$ $<0$, each $y_{1, y}(t)>0$ and each $y_{1, j}^{\prime}(t)<0$ for all $t$.

Suppose that $y_{1}(t)$ is positive and exceeds $\alpha+$ $x_{1}(t)$. If $x_{1}(t) \leqslant 0$ then $y_{1}$ gets only positive summands at moment $t$. If $x_{1}(t)>0$ then

$y_{1}(t+1) \geqslant y_{1}(t)-\alpha>x_{1}(t)>0$.

In any case, $y_{1}(t+1)>0$. It remains to check that $y_{1}(t+1)>\alpha+x_{1}(t+1)$. First suppose that $x_{1}(t)$ $>0$. Then

$$
\begin{aligned}
y_{1}(t+1) & =y_{1}(t)+\beta^{\prime}(h+1)-\alpha \\
& >\alpha+x_{1}(t)+\beta^{\prime}(h+1)-\alpha \\
& \geqslant \alpha+x_{1}(t+1) .
\end{aligned}
$$

The case $x_{1}(t)=0$ is trivial: $x_{1}$ does not change at moment $t$ whereas $y_{1}$ increases. So suppose that $x_{1}(t)<0$. Then

$$
\begin{aligned}
& y_{1}(t+1)=y_{1}(t)+\beta^{\prime}(h+1)+\alpha^{\prime}, \\
& x_{1}(t+1) \leqslant x_{1}(t)+\beta-\beta^{\prime} h-\alpha^{\prime} .
\end{aligned}
$$

By the choice of $h$, the increase of $y_{1}$ is at least as big as the increase of $x_{1}$. The lemma is proved.

Proof of Theorem 3.1 (continued). There exists a positive probability $\varepsilon$ that all conditions of the first (resp. second) part of the lemma are satisfied for a given $u$ (resp. $v$ ). With probability $(1-(1-$ $\left.\varepsilon)^{n / 3}\right)^{2}$ there exist $u, v$ such that the conditions of both parts of the lemma are satisfied. But the conclusions of the lemma imply that no $s(t)$ satisfies $B$ and therefore no $s(t)$ satisfies $C$.

\section{References}

[1] V.I. Arnold, Ordinary Differential Equations (Nauka, Moscow, 1975; in Russian).

[2] Y.V. Matijasevitch, Possible nontraditional methods of establishing unsatisfiability of propositional formulas. In: D.A. Pospelov, G.E. Mints and R.I. Freidzon, eds., Questions of Cybernetics: Problems of Reducing the Exhaustive Search (Soviet Academy of Sciences, Moscow, 1987; in Russian).

[3] P.W. Purdom and C.A. Brown, Polynomial-average-time satisfiability problems, Inform. Sci. 41 (1987) 23-42. 